\title{
THE METHODOLOGY OF THE BIOMECHANICAL MOVEMENTS FOR THE DISABLED BASED ON ART THERAPY
}

The essence of the research work is to open up the new possibilities in the treatment processes within rehabilitation, physiotherapy and consequent movement re-education process for people with disabilities, post-traumatic conditions, or degenerative disease. Art therapy is a special type of psychotherapy that focuses on the development of creative skills, emotional development and interpersonal relationships of the mentally, physically or socially disturbed individuals. The methodology proposal includes the systems of rehabilitation, therapeutic physical education, physiotherapy, and ergotherapy. The purpose of the methodology of the biomechanical movements using art therapy is to follow and build on the classic rehabilitation of the patients with physical disabilities. The aim of this form of therapy is to increase the strength of weakened muscles and the range of joint movement, to improve the neuromuscular coordination, and to improve the physical independence of the physically disabled individuals.

Keywords: Art therapy, motor and sensory functions, biomechanical movements, artistic means of expression, biomechanical principles, methodology, biomechanics of joints and systems, rehabilitation, physiotherapy, physical disability.

\section{Introduction}

The biomechanical principles of a healthy individual's momentum form the basis for the work with the physically disabled clients. The doctor, the physiotherapist, the rehabilitation worker and the biomechanics specialist treat and help the patient on the basis of their knowledge of anatomy and the biomechanics of the human body. From their experience it is known that the disabled need psychological support, a strong incentive to handle the rehabilitation and then the integration into everyday life. As a relatively young discipline, the art therapy has good results in the field of psychology and psychiatry. It forms a bridge to the mental balance of the clients and their motivation to live. By combining biomechanical principles and art techniques we can form a methodological foundation for the therapy improving the condition of damaged segments of the human body. The cooperation of the physiotherapist and the art therapist has the potential to start a new active form of rehabilitation, suitable for clients with permanent physical damage or a post- traumatic condition. The art therapy and the biomechanics may seem different but these two progressive disciplines united in one unit create the new therapy methodology for the disabled.

\section{The purpose of the methodology} of the biomechanical movements based on art therapy

The purpose of the methodology is to correctly combine the biomechanical movements according to the ideal biomechanical parameterization of the motions of the individual segments of the human body. Through the art therapy techniques, the aim is to find the ideal approach to the client, to gain his/her interest and subsequently motivate him/her to an independent and active attitude. The active attitude and the positive emotions of the client while applying the methodology have a great importance from both qualitative and quantitative points of view. Art therapy is useful in training and maintaining motor and sensory functions. Art therapy interventions are used for example with people after a stroke in order to exercise the fine and gross motor skills, to restore the memory functions and to maintain the emotional stability [1].

\footnotetext{
* 'Ivana Zidek, ${ }^{2}$ Jana Musinska, ${ }^{2}$ Jozef Zivcak

${ }^{1}$ Faculty of Arts, University of Presov, Slovakia

${ }^{2}$ Department of Biomedical Engineering and Measurement, Faculty of Mechanical Engineering, Technical University, Kosice, Slovakia

E-mail: jozef.zivcak@tuke.sk
} 


\section{The definition of the art therapy and the forms and techniques used in the methodology of the biomechanical principles}

It is important to determine if the client has the skills of artistic expression appropriate to his/her age and disability. Art therapy is applied as a clinical form of therapy or as a diagnostic method where it is possible to diagnose the client using the special drawing tests. The drawing enables us to determine the level of intellectual abilities, the extent of motor skills damage, the extent of psych -motor abilities; it helps to find the signs of organicity, or visual motor coordination impairments.

The most common techniques include a finger-painting, drawing together with the therapist, drawing together with the group, textile forms, working with stone, wall painting and working with clay. With the art therapy we use one or more art techniques, working either on some surface (e.g.: painting with watercolours or tempera paint, collage, or work with paper), or three dimensions (e.g. modelling, pottery, woodwork). Activities include the technique of doodling, freehand drawing or painting, thematic drawing, painting of dreams, fantasies, desires and memories, self-portraits and portraits. The diversity of creative techniques and activities is therefore suitable to raise self-esteem, even with those clients who are not too skilled in drawing and painting [2].

\section{The classification of the patient and the procedures used}

In this research work, the methodology and algorithms for disabled patients are proposed. The most common types of disability that can be helped through art therapy include: cerebral palsy (deficiency of motor control and momentum development caused by brain damage at the time before birth, at birth or within 1 year after the birth), inflammatory brain diseases, epilepsy, mild polio, amputations, myopathy (progressive muscular dystrophy), curvature of the spine disorders, congenital developmental disorders. Another group is formed by patients with the failing vital functions but with retained consciousness and physical activity (respiratory distress, circulatory failure, etc.). In the beginning, only passive movements are practiced, gradually the active movements are increased, and then sitting, standing and walking are practiced [3 and 4].

The standard procedures of kinesiology focused on physiotherapist's indicative potential are necessary for building the foundation of the biomechanical principles methodology. Patient or client is at first examined in order to compare the mobility of the joint and the strength of muscle with the healthy limb. According to the observed results, a gradual re-education of the affected part's own function is determined. From the methods of kinesiology, especially muscle strengthening and the limb's re-education are used. All kinds of movements from kinesiology (controlled motion, pendulum, swing, and pulling motion) are applied with the methodology using art therapy depending on the client's disability following the professional consultation with a doctor and a physiotherapist. The contraindications must be taken into account, especially with swing motions with the inflammatory process in the joint. Before initiating therapy, the analysis and synthesis of movements is made and according to the results, the methodological process is adjusted so that the exercise is adequate and does not cause fatigue, or poor coordination due to wrong selection of the methodology. Compensating the disability of one segment by using the muscles of a healthy segment in substitution while performing a particular movement must be avoided at the therapy [5, 6 and 7].

With the treatment using the methodology of biomechanical movements through art therapy, large areas are usually chosen, such as boards, paper pack hanging on the wall or laid out spread on the floor. Soft and fine painting techniques are preferred. The width and intensity of the paint trail indicate the pressure and the stability of the limb. Water-diluted colors are deemed best, from both sanitary and health point of view. They do not contain any volatile chemicals that could harm the health of a client if inhaled. At the end of the activity, they are easily removable from the surface. Paper as a base material is deemed best because of its capacity to absorb water, it's basically a blotting material. The painting dries quickly enough and leaves the informative trail of the limb.

\section{The biomechanics of the elbow joint}

From the functional point of view it consists of the ulnohumeral hinge joint, the radiohumeral ball joint and the proximal radioulnar joint. Considering their configuration, two types of motion can occur in the elbow joint independently of each other, the flexion-extension and supination-pronation. The flexion-extension movement occurs in the ulnohumeral and radiohumeral joint (Fig. 1). From the kinematics' and stability's point of view, the shape of joint planes of ulnohumeral joint is of critical importance. From the practical point of view, this movement is considered uniaxial. The axis is more or less equivalent to the axis of the humeral trochlea. The full range of the motion ranges from $125^{\circ}$ to $145^{\circ}$. The range of hyperextension is minimal, because it is blocked by the tension in the frontal part of the socket and by the olecranon summit contacting the fossa. Pronation-supination occurs in the elbow, simultaneously in the radiohumeral, proximal radioulnar and distal radioulnar joint (Fig. 1). With this movement, the relative position of radius and ulna is changed, therefore causing the change of palm orientation. According to the classic description, the radius rotates around the ulna which does not change its position. This statement is not 
entirely correct; the supine-prone motion, especially in the distal radioulnar joint, is quite complicated.

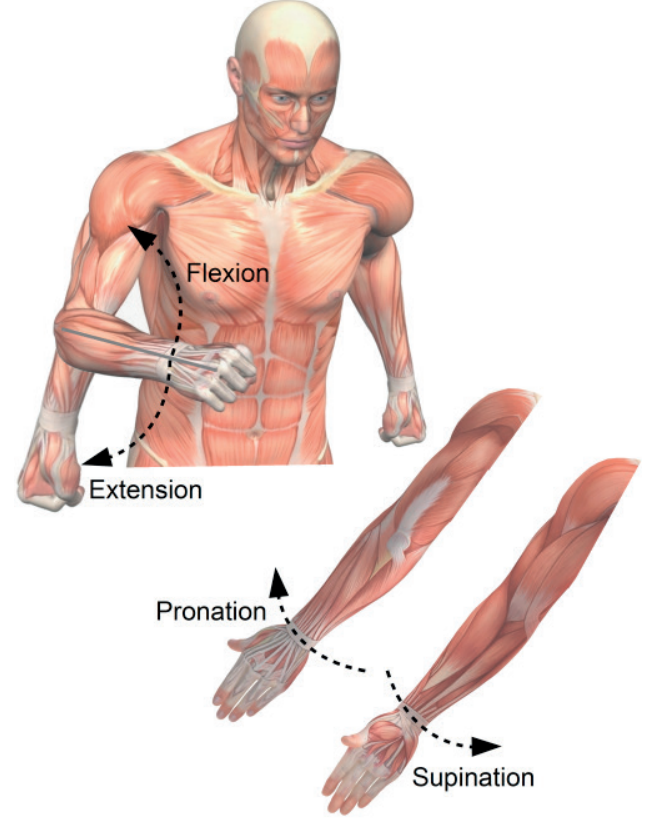

Fig. 1 The scheme of the biomechanics of the elbow joint

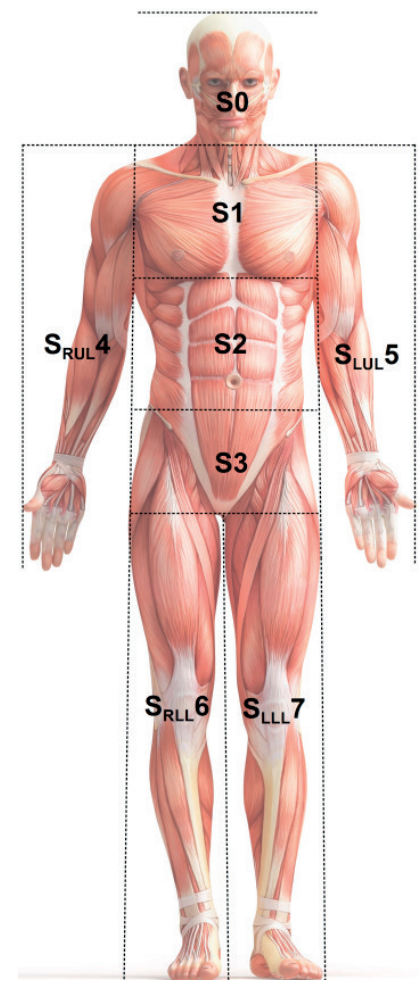

$\mathbf{S 0}$ - Sector of the head, $\mathbf{S} \mathbf{1}$ - Sector of the thorax, $\boldsymbol{S} 2$ - Sector of the separate organs, $\mathbf{S} 3$ - Sector of the pelvis, $\mathbf{S} 4$ - Sector of the right upper limb, S5 - Sector of the left upper limb, S6 - Sector of the right lower limb, S7- Sector of the left lower limb,

Fig. 2 Scheme of the layout of the sectors of the human body

\section{The biomechanical parameterization and graphic scheme}

Depicting the layout of the human body sectors with the indication of the standard range of joint movements is used to schematically illustrate the biomechanical parameterization (Fig. 2). The following pictures illustrating the individual methodical procedures show the parameterization of the biomechanical movements indicating the range of movement.

7. The methodology of the biomechanical principles with the malfunctions of the elbow joint (MBPEJ)

The biggest problem with the damage to the elbow joint is flexion and extension. As mentioned above, the movement of the flexion and extension is uniaxial and the full range of movement of the healthy elbow joint ranges from $125^{\circ}$ to $145^{\circ}$ (Fig. 3A). The
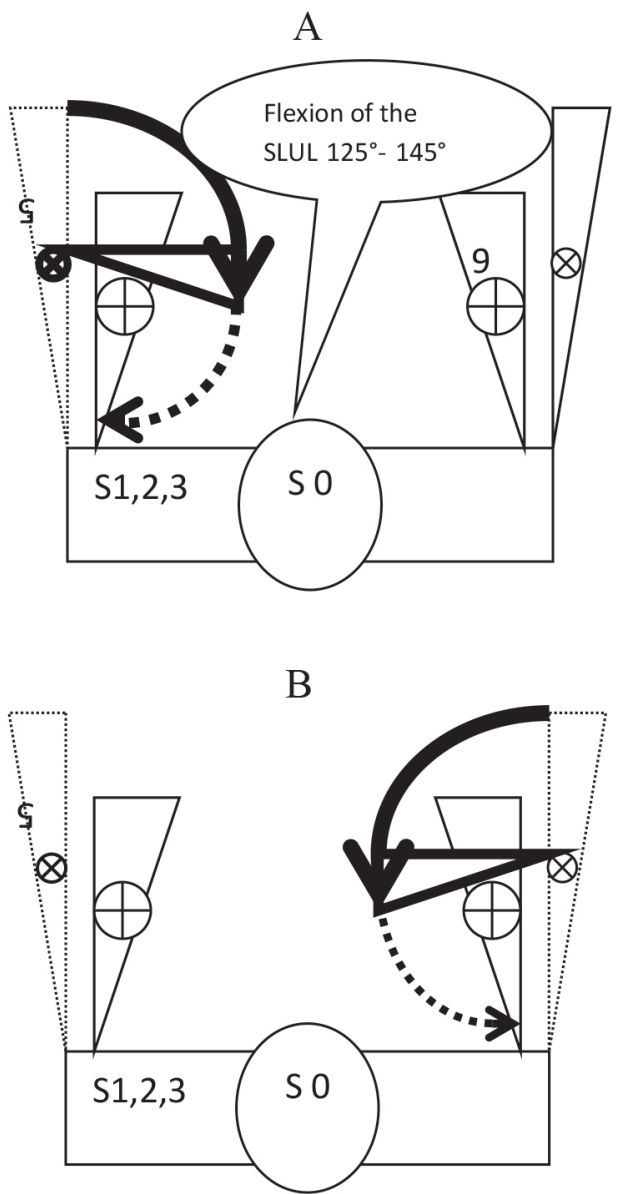

Fig. 3 Image of the flexion $A$ in the elbow joint of the left upper limb 5 SLUL, standard range of movement up to $125^{\circ}$ or maximum $145^{\circ}$, $B$ standard range of movement of the 4 SRUL in the shoulder joint, adduction $135^{\circ}$, the position of sitting, standing. 
range of hyperextension is minimal while the flexion is limited and, with more muscular individuals, affected by the muscles of arm and forearm. Serious damage, possibly with an oedema, swelling and reddening in the inflamed area is dealt with noninvasively, by focusing on the arm and wrist. The elbow joint in the acute condition is immobilized and put into a sling. The technique of finger-painting is used to exercise the fine motor skills of the hand, at the minimal movement in the elbow joint. For the supination and pronation, it is most advisable to use the technique of pressing stamps on the pad, eventually with the gradual positive effect and improved condition, we can move on to the technique of imprinting into soft material.

Seated position: The working pad is in the horizontal position; the elbow can be immobilized or supported. The art therapist actively hands the patients various kinds of stamps, varying their size. It is important to hand them in a specific way so that the palm is in supination and turning fluently into pronation while dipping the stamp into the paint. The stamp absorbs the liquid paint while moving in a circular motion and then by putting pressure on the paper pad it creates a picture. The art therapist proceeds this way, with the sporadic breaks, during which the elbow, if not immobilized, can be put into the position of ease in the sling (Fig. 3B).

Standing position: In the case of the immobilization of the arm, to prevent the compensating movements (when the patient unconsciously substitutes the damaged segment by using the healthy segment while performing a particular movement), the supine and prone element together with the flexion and extension are best strengthened in standing position. The paper is placed on the wall; the paints are placed on the pad at the height of the flexed elbow. Stamps can also be used. By painting on the wall with a soft brush, the patient strengthens the flexion and extension with downward and upward strokes. After the improvement of the condition, the arm is not immobilized, the range of movements is increased and the patients use the muscles of the arm, elbow and wrist together. The paint brush with tougher bristles is selected for the very reason of exercising stronger pressure on the paper pad.

To illustrate with an example, a brief case is presented of the 53-year old female patient with a Sjogren's syndrome diagnosed in 1983, suffering from myeloradiculitis with combined quadriparesis. The patient is single, childless and in the care of the retirement house. She moves with the aid of a wheelchair, partially independent, position active, speech articulate, strength symmetrical, she can stand when supported, unable to walk. Limited movement in left arm in all directions, elbows, wrists and small hand joints are free without the signs of arthritis, fine motor skills preserved, grip slightly weakened, she does not cite joint pain. Lower limbs: muscle atrophy of both upper limbs, active flexion of both lower limbs up to $90^{\circ}$, without the signs of arthritis, serious neuropathy of the lower limbs with the dysesthesias (Fig. 4).

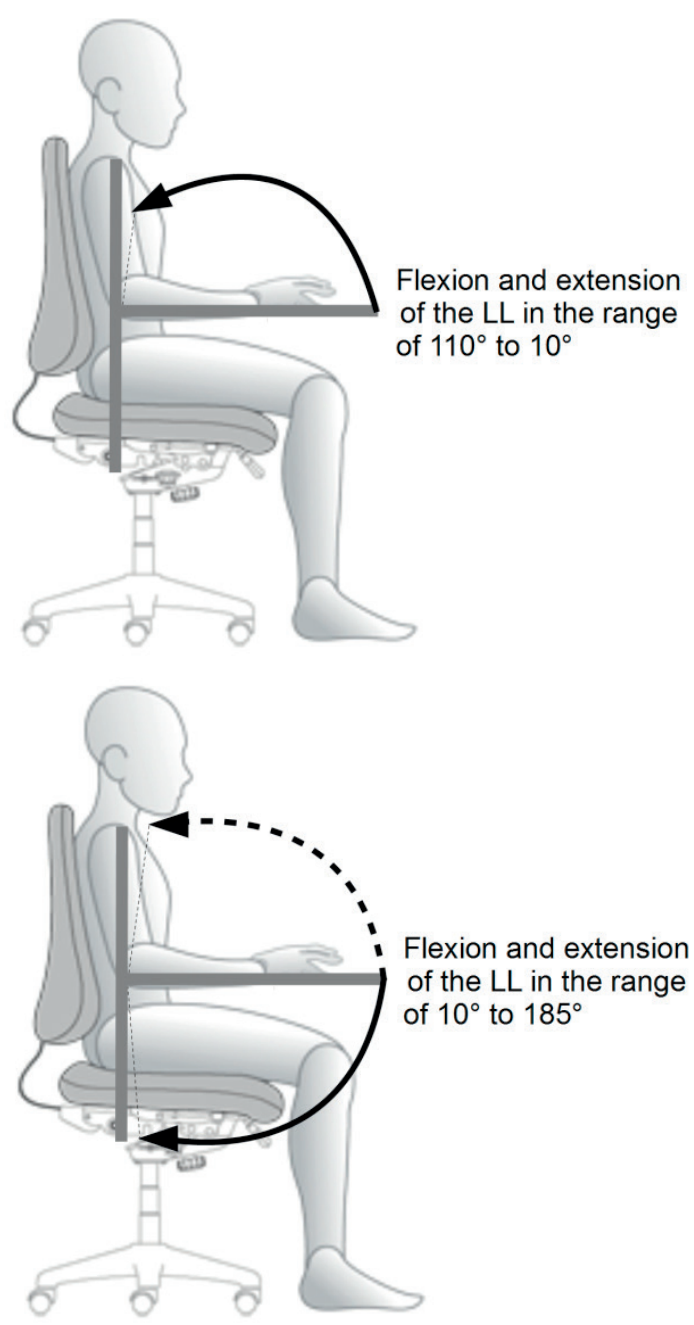

Fig. 4 Image of the flexion of the elbow joint at the standard range of movement from $10^{\circ}$ to $185^{\circ}$, the seated position

Plan of implementation of the Methodology of the biomechanical motions:

The methodology was applied in the order MBMSJ (shoulder joint), MBMEJ (elbow joint), MBMFA (forearm) in the time sequence and relative dependence according to the degree of results achieved and considering the pain and fatigue demonstrated by the respondent.

\section{The methodology of the biomechanical principles with the malfunctions of the elbow joint (MBPEJ)}

Although the respondent does not show any signs of increased damage of the elbow joint, the most common procedures from this methodology were used. Specifically it was necessary to take into consideration the individual aspects of the respondent in each 
case. Particular complications occur due to the Sjogren syndrome, the missing sensitivity in the right upper limb. The respondent is not able to perceive without full concentration the releasing and falling of the arm from the position of $90^{\circ}$ flexion, for instance when the right upper limb is placed on the wheelchair's armrest. For that reason we exercise the elbow joint but especially the muscles of the forearm. The movement to flexion is more fluent in the range up to $90^{\circ}$ of incomplete extension, approximately $10^{\circ}$. The flexion above $90^{\circ}$ is considerably problematic, uncoordinated and not sufficiently fluent.

Seated position: the working pad is put into horizontal position, adjustable for setting and tilting under such angle and direction as the respondent needs. The pad is made of light material with round edges for safety reasons. The elbows are not immobilized, the right upper limb is exercised more, and it is also more pressured with better function of the arm, humerus osteolysis present. Paper is firmly attached to the pad to record the movements of the forearm, wrists and hands of the respondent. Various techniques were used, most frequently the drawing with colored markers - suitable with the insufficient ability of elbow flexion and diminished function of the group of muscles of the arm. When forced to put pressure on the arm and arm muscles, the respondent is forced to use the compensating movement of group of muscles of the nape, neck and upper back part, which results in her tiring fast and easily. This fact is crucial also for the selection of the pad. It is preferred to be horizontal, possible tilted with the option of free slide.

The result is improved coordination ability of both upper limbs, with subsequently decreased simultaneous movement of the whole torso, the movements of the left upper limb are more fluent and the range of movement of both upper limbs improved by $15^{\circ}$ on average.

During the implementation of the project it was concluded that the key to help the physically handicapped lies in their own active approach. Clients' self-reflection and motivation lead to significant improvement of the mobility of their physical segments. This is confirmed not only by the statistical evaluation of the anonymous questionnaires and measurements in the experimental and control group of clients, but also individual case and the measurements by the SFTR method (Table 1). The vision of the project, as it turned out during the implementation, is the creation of the single computer program compatible with the interactive board.

\section{Conclusion}

The handicapped have a whole range of creative possibilities as far as their physical and mental conditions allow. Art therapy has the potential not only for the rehabilitation of fine motor skills but also for compensation of the mental handicap. To experience success, to encourage one's self-confidence by one's own activities, the sense of creative achievement, it all helps. Art therapy can be applied in various situations and with various diagnoses. The aim of the biomechanical algorithms is to focus on the biomechanical aspects and structures of the human body. The biomechanical perspective and the biomechanical approach are also the focus in several scientific disciplines and professional

Table of examination by the SFTR method

Table 1

\begin{tabular}{|c|c|c|c|c|}
\hline PRETEST & Shoulder joint & Elbow joint & Forearm & Wrist joint \\
\hline Sagittal RUL & $30-0-90^{\circ}$ & $0-0-120^{\circ}$ & $90-0-80^{\circ}$ & $50-0-40^{\circ}$ \\
\hline Frontal RUL & $60-0-25^{\circ}$ & & & $25-0-40^{\circ}$ \\
\hline Transverse RUL & $20-0-95^{\circ}$ & & & \\
\hline Rotating RUL & $70-0-60^{\circ}$ & & & \\
\hline Sagittal LUL & $20-0-50^{\circ}$ & $0-0-120^{\circ}$ & $70-0-80^{\circ}$ & $40-0-40^{\circ}$ \\
\hline Frontal LUL & $30-0-0^{\circ}$ & & & $20-0-40^{\circ}$ \\
\hline Transverse LUL & $20-0-10^{\circ}$ & & & \\
\hline Rotating LUL & $0-0-0^{\circ}$ & & & \\
\hline POSTTEST & Shoulder joint & Elbow joint & Forearm & Wrist joint \\
\hline Sagittal RUL & $30-0-90^{\circ}$ & $0-0-135^{\circ}$ & $90-0-80^{\circ}$ & $50-0-50^{\circ}$ \\
\hline Frontal RUL & $70-0-30^{\circ}$ & & & $25-0-45^{\circ}$ \\
\hline Transverse RUL & $20-0-100^{\circ}$ & & & \\
\hline Rotating RUL & $70-0-70^{\circ}$ & & & \\
\hline Sagittal LUL & $20-0-50^{\circ}$ & $0-0-135^{\circ}$ & $70-0-80^{\circ}$ & $40-0-40^{\circ}$ \\
\hline Frontal LUL & $30-0-0^{\circ}$ & & & $25-0-50^{\circ}$ \\
\hline Transverse LUL & $20-0-10^{\circ}$ & & & \\
\hline Rotating LUL & $0-0-0^{\circ}$ & & & \\
\hline
\end{tabular}


fields. Biomechanics in collaboration with art therapy has a wide range of possibilities in terms of rehabilitation of the human body, from each individual's active approach. The methodology of the biomechanical motion helps to understand the context of the biomechanical movements and using the art therapy techniques, it helps to implement their proper use.

The proposal of the methodology of the biomechanical movements and its subsequent application in the rehabilitation processes in practice, together with physical therapy, will be an important step in the progress of these disciplines. It will significantly change the current view of the patient as well as the patient's attitude to the therapy of his/her body.

\section{Acknowledgement}

This study was supported by the grant VEGA $1 / 0515 / 13$ of the Ministry of Education of the Slovak Republic

\section{References}

[1] GARNER, R. L.: Factors in Neuropsychological Art Therapy. American J. of Art Therapy, vol. 34, No. 4, 1996, p. 1-6.

[2] FABRICI, J.: Basics of Artheterapy (in Czech), Praha, 2002. 176 p.

[3] GUTH, A. et al.: Diagnostic Methods for Physiotherapists (in Slovak), Bratislava, 2004. 400 p.

[4] HROMADKOVA, J. et al.: Physiotherapy (in Czech), Jinocany: H\& H, 2002. 428 p.

[5] ZIVCAK, J., KNEZO, D.: Biomechanics of Momentum (in Slovak), Presovska univerzita: Presov, 2008. 208 p.

[6] BARTONICEK, J., HERT, J.: Basics of Clinical Anatomy of Movement Apparatus (in Czech), Praha, 2004. 256 p.

[7] DYLEVSKY, I.: Kinesiology - Basics of Structural Kinesiology (in Czech), Praha, 2009. 235 p. 